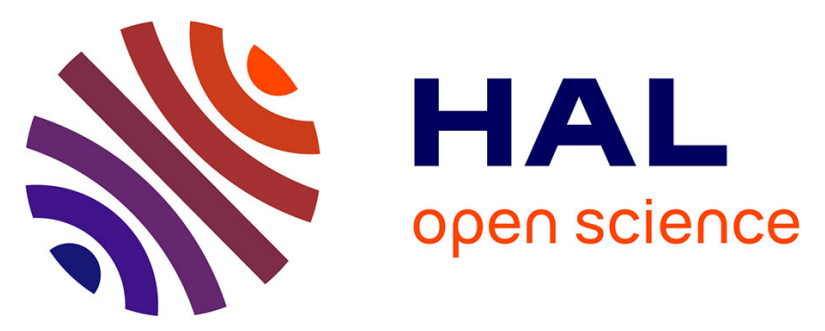

\title{
Pacing strategy during the initial phase of the run in triathlon: influence on overall performance
}

Christophe Hausswirth, Yann Le Meur, François Bieuzen, Jeanick Brisswalter, Thierry Bernard

\section{To cite this version:}

Christophe Hausswirth, Yann Le Meur, François Bieuzen, Jeanick Brisswalter, Thierry Bernard. Pacing strategy during the initial phase of the run in triathlon: influence on overall performance. European Journal of Applied Physiology, 2010, 108 (6), pp.1115-1123. 10.1007/s00421-009-1322-0 . hal-01724323

\section{HAL Id: hal-01724323 \\ https://hal-insep.archives-ouvertes.fr/hal-01724323}

Submitted on 6 Mar 2018

HAL is a multi-disciplinary open access archive for the deposit and dissemination of scientific research documents, whether they are published or not. The documents may come from teaching and research institutions in France or abroad, or from public or private research centers.
L'archive ouverte pluridisciplinaire HAL, est destinée au dépôt et à la diffusion de documents scientifiques de niveau recherche, publiés ou non, émanant des établissements d'enseignement et de recherche français ou étrangers, des laboratoires publics ou privés. 
article publié dans : European journal of applied physiology (ISSN 1439-6319), 2010, vol. 108,
$\mathrm{n}^{\circ} 6$, pp. 1115-1123 DOI : $10.1007 / \mathrm{s} 00421-009-1322-0 \quad$ PMID 20024576

TITLE: PACING STRATEGY DURING THE INITIAL PHASE OF THE RUN IN TRIATHLON: INFLUENCE ON OVERALL PERFORMANCE

AUTHORS: CHRISTOPHE HAUSSWIRTH ${ }^{1 *}$, YANN LE MEUR ${ }^{1,2}$, FRANCOIS BIEUZEN $^{1}$, JEANICK BRISSWALTER ${ }^{2}$, THIERRY BERNARD 3

${ }^{1}$ Research Department, Laboratory of Biomechanics and Physiology, National Institute of Sport and Physical Education, 75012 Paris, FRANCE.

${ }^{2}$ Laboratory of Physiological Adaptations, Motor Performance and Health (EA 3837), University of Nice-Sophia Antipolis, Faculty of Sport Sciences, BP 32 59, 06205 Nice Cedex 03, France.

${ }^{3}$ Sport Ergonomy and Performance Laboratory, HANDIBIO EA 3162, University of Sud Toulon-Var, BP 132, 83957 La Garde Cedex, France.

*Corresponding Author :

Dr Christophe Hausswirth, $\mathrm{PhD}$

Research Department,

National Institute of the Sport and Physical Education (INSEP),

11 Avenue du Tremblay, 75012 Paris, FRANCE

Phone : + 33 1-41-74-43-85

Fax : + 33 1-41-74-45-35

E-mail : christophe.hausswirth@insep.fr 


\section{ABSTRACT}

2 Objective: The aim of the present study was to determine the best pacing strategy to adopt during the initial phase of a short distance triathlon run for highly-trained triathletes.

Methods: Ten highly-trained male triathletes completed an incremental running test to determine maximal oxygen uptake, a 10-km control run at free pace and three individual timetrial triathlons (1.5-km swimming, 40-km cycling, 10-km running) in a randomised order. Swimming and cycling speeds were imposed as identical to the first triathlon performed and the first run kilometre was done alternatively $5 \%$ faster (Tri-Run $+5 \%$ ), $5 \%$ slower (Tri-Run $-5 \%$ ) and $10 \%$ slower (Tri-Run $_{-10 \%}$ ) than the control run (C-Run). The subjects were instructed to finish the 9 remaining kilometres as quickly as possible at a free self-pace.

Results: Tri-Run-5\% resulted in a significantly faster overall 10 -km performance than TriRun $_{+5 \%}$ and Tri-Run-10\% $(p<0.05)$ but no significant difference was observed with C-Run

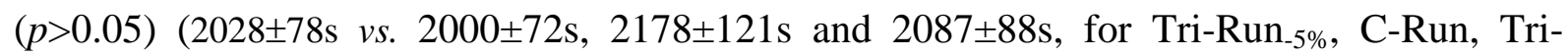
Run $_{+5 \%}$ and Tri-Run $-10 \%$, respectively). Tri-Run $+5 \%$ strategy elicited higher values for oxygen uptake, ventilation, heart rate and blood lactate at the end of the first kilometre than the three other conditions. After 5 and 9.5 kilometres, these values were higher for Tri-Run-5\% $(p<0.05)$.

Conclusions: The present results showed that the running speed achieved during the cycle-torun transition is crucial for the improvement of the running phase as a whole. Triathletes would benefit to automate a pace $5 \%$ slower than their $10-\mathrm{km}$ control running speed as both $5 \%$ faster and $10 \%$ slower running speeds over the first kilometre involved weaker overall performances.

Keywords: Triathletes, Pace, Running speed, Long duration exercise, Fatigue, Central governor model, Previous experience 
The Olympic distance triathlon (i.e. short distance triathlon) is a unique effort, which involves successively $1500 \mathrm{~m}$ swimming, 40-km cycling and 10-km running. The ability to link the three triathlon disciplines in an optimal manner has been described as an important determinant of success (Bentley et al. 2002, Hausswirth and Brisswalter 2008). This observation is even more relevant for the cycle-to-run transition as all the recent studies conducted during ITU World Cup triathlon competitions (i.e. short distance triathlon) have reported high correlation between finish position and running performance for both genders (coefficients of correlation ranging from 0.71 to $0.99, p<0.01$ ) (Vleck et al. 2006, Vleck et al. 2008; Le Meur et al. 2009). These coefficients of correlation were significantly lower considering overall ranking and both swimming performance (from 0.36 to $0.52, p<0.01$ ) and cycling performance (from "no significant correlation" to 0.74, $p<0.05$ ) (Vleck et al. 2006, Vleck et al. 2008, Le Meur et al. 2009).

In this context, several studies have focused on strategies for improving the performance during the triathlon run. These studies have identified drafting position (Hausswirth et al. 1999), variability in cycling power output production (Bernard et al. 2007), cycling cadence selection (Gotschall et al. 2002, Vercruyssen et al. 2005) and previous locomotion mode (Hausswirth et al. 1996, Hausswirth et al. 1997) as the main determining factors of performance. On the other hand, less attention has been given to identify the best pacing strategy to adopt over the running leg. Only Kreider et al. (1988) showed that a progressive increase in running pace during the onset of the triathlon run allows the attainment of a 
ventilatory, cardiovascular, and neuromuscular steady-state. Recent studies have reported that triathletes tend to adopt a positive pacing during the run phase of ITU World Cup races, whereby after that a peak speed was reached, triathletes progressively slowed down (Vleck et al. 2006, Vleck et al. 2008, Le Meur et al. 2009). During 2001 and 2002 Lausanne World Cup most athletes ran faster over the first kilometre than most other run sections (Vleck et al. 2006, Vleck et al. 2008), while residual effects of prior cycling are the highest and despite the recommendations of the current literature to adopt an even pacing strategy (i.e. constant pace) for physical events of such duration (for a review, see Abbiss and Laursen 2008). Similarly, Le Meur et al. (2009) showed that all of the 136 triathletes competing in the 2007 Beijing ITU WC event adopted a "positive pacing strategy” (whereby speed gradually declines, Abbiss and Laursen 2008) through the running phase. During this race, the first of the four laps was run $10.0 \%$ faster than the three remaining laps.

Accordingly, we hypothesised that a positive pacing during the running phase of a short distance triathlon is the best strategy to achieve the best overall performance for highly trained triathletes. The aim of the present investigation was to compare the effectiveness of three different pacing strategies during the initial phase of a $10-\mathrm{km}$ triathlon run, while respecting normal triathlon conditions. As the transition from cycling to running represents the most critical and strategic phase effecting finish position, we investigated the effects of the pace adopted over the first run kilometre on the overall triathlon performance. 


\section{Participants}

Ten well-motivated male triathletes currently competing at a national level and selected on the basis of their performance time over the short distance triathlon (2h02 $\pm 7 \mathrm{~min}$ ) volunteered to take part in this experiment. They had trained regularly and competed in triathlons for at least four years. Their characteristics are presented in Table 1. The triathletes were fully informed of the content of the experiment, and written consent was obtained before any testing, according to local ethical committee guidelines (Saint Germain en Laye, France). To familiarize the triathletes with the cycling and running circuits used in the experiment, a training camp was programmed 10 days before with a light training program. During the entire experimental procedure, the subjects did not perform any exhausting exercise in the 48 hours preceding each test.

\section{Maximal running test}

Prior to the experiment, each subject underwent a running test to determine maximal oxygen uptake ( $\dot{\mathrm{V}} \mathrm{O}_{2 \max }$ ) and ventilatory thresholds (VT1, VT2) on a track where the increment of speed was fixed at $1 \mathrm{~km} \cdot \mathrm{h}^{-1}$ each 3 minutes. Oxygen uptake $\left(\dot{\mathrm{V}} \mathrm{O}_{2}\right)$ and expiratory flow $\left(\dot{\mathrm{V}}_{\mathrm{E}}\right)$ were recorded breath by breath with a telemetric gas exchange measurement system (Cosmed K4b², Rome, Italy). Heart rate values (HR) were monitored every second using a Polar unit (RS800sd, Polar Electro, Kempele, Finland). Expired gases and HR values were subsequently averaged every 5 s. $\dot{\mathrm{V}} \mathrm{O}_{2 \max }$ was determined according to criteria described by Howley et al. 
1 (1995) - that is, a plateau in $\dot{\mathrm{V}} \mathrm{O}_{2}$ despite an increase in running speed, a respiratory

2 exchange ratio value of 1.15 , or a Heart rate (HR) over $90 \%$ of the predicted maximal HR.

$3 \dot{\mathrm{V}} \mathrm{O}_{2 \max }$ was then determined as the highest value of $\dot{\mathrm{V}} \mathrm{O}_{2}$ achieved during a period of 30-sec.

4 The first and the second ventilatory thresholds (VT1 and VT2, respectively) were determined

5 according to criteria previously described by Beaver et al. (1986). VT1 was determined as the

6 first breakpoint where we detected a systematic increase in $\dot{\mathrm{V}}_{\mathrm{E}} / \dot{\mathrm{V}}_{2}$ without a concomitant

7 increase in $\dot{\mathrm{V}}_{\mathrm{E}} / \dot{\mathrm{V} C O}_{2}$. VT2 was associated with the first breakpoint detected where $\dot{\mathrm{V}}_{\mathrm{E}} / \dot{\mathrm{V}}_{\mathrm{CO}}$

8 started to increase concomitantly with $\dot{\mathrm{V}}_{\mathrm{E}} / \dot{\mathrm{V}} \mathrm{O}_{2}$.

\section{Control run}

The first test was a 10-km run performed on a 340-m indoor running track (control run, CRun). Pacing strategy was left free and the only instruction given to the triathletes was to run as fast as possible over the $10-\mathrm{km}$. No-feedback was given about running speeds or split times. Subjects were informed of each kilometre completed. They had the possibility to drink $250 \mathrm{~mL}$ of water at the end of the $3^{\text {rd }}$, the $6^{\text {th }}$ and the $9^{\text {th }}$ kilometre.

\section{The three triathlon sessions}

All experiments (Fig. 1) were carried out in Paris, specifically at the French National Institute of Sport and Physical Education (I.N.S.E.P.) from January to March. The study was conducted on indoor cycling and running tracks. Inside air temperatures ranged from $18^{\circ}$ to $20^{\circ} \mathrm{C}$. The three experimental triathlons were performed alone (i.e. time-trial triathlons) in a randomized order over the short distance (1.5-km swim, 40-km bike, 10-km run) with a 10- 
day recovery between them, where training load was minutely controlled. Heart rate at ventilatory thresholds (VT1, VT2) identified during the maximal running test were used to demarcate 3 intensity zones (Esteve-Lanao et al. 2007). These included zone 1, low-intensity exercise performed below VT1; zone 2, moderately high-intensity exercise in an intensity range between VT1 and VT2; and zone 3, high-intensity aerobic exercise performed above VT2. In the month prior to the first experimental trial, training durations and distributions of time spent in the three intensity zones were continuously monitored (15 \pm 3 h.wk ${ }^{-1}$ and $79 \%$ $10 \%-12 \%$, respectively). The mean training load was similar to previous data reported in the literature for trained triathletes (Hausswirth et al. 1997), high-level runners (Esteve-Lanao 2007), elite rowers (Fiskestrand and Seiler 2004) and cross-country skiers (Seiler and Kjerland 2006). Throughout the entire experiment all subjects were coached by the same person. Training load was controlled to be similar in the duration between each test and similar to the participants usual training pattern. Triathletes were restricted to train in zone 1 during each couple of days following or preceding each test. To avoid injuries or overreaching, daily feedback was also obtained from triathletes and taken into account.

Swimming-cycling phase. The swim was staged in an indoor $50-\mathrm{m}$ pool $\left(24-25^{\circ} \mathrm{C}\right)$ wearing a singlet. The 40-km bike segment was conducted on a cycling track (166m) next to the pool. The swim-cycle combination was performed in the three experiments at the same speed as the first triathlon, which was completed as fast as possible. The swimming velocity was controlled using a pacer placed in the swimming cap (Tempo Trainer, Finis ${ }^{\circledR}$, Helsinki, Finland), which provided a ring signal each period of time needed for the completion of 12.5m. During the first triathlon, they were asked to swim with an even paced strategy. During the first 3-km of the bike, triathletes had to reach the speed to be maintained during the last 37$\mathrm{km}$. A ring signal at each half-lap (83m) indicated precisely the speed they had to keep. The 
1 speed of the last 37-km was the one reached from the second to the third kilometre,

2 considering therefore that the two first kilometres was the distance necessary to reach a constant speed (Hausswirth et al. 2001). During the cycling sections, triathletes could drink ad libitum thanks to $750 \mathrm{~mL}$ water-bottles disposed on their bikes. The transition time between swimming-cycling was slightly different from those obtained in competition (i.e. 3 minutes). It included one minute for a change of clothes, one minute for the cardiofrequency meter installation on the subject and one minute for the run with the bike to reach the cycling track (200m).

Running phase. The 10-km run was staged next to the cycling track, on the same indoor synthetic running track as the C-Run (340m). During the first kilometre, subjects had to maintain alternatively a running speed 5\% faster (Tri-Run $+5 \%$ ), 5\% slower (Tri-Run $-5 \%$ ) and 10\% slower (Tri-Run-10\%) than the mean speed of the C-Run. The subjects were then instructed to finish the 9 remaining kilometres as quickly as possible, as in a competitive event. Tri-Run $+5 \%$ condition was representative of the strategy adopted by highly-trained triathletes in competition during the cycle-to-run transition (Le Meur et al. 2009). Tri-Run-5\% and Tri-Run-10\% were closer to the mean velocity they used to maintain during short distance triathlon. A ring signal each $25 \mathrm{~m}$ indicated precisely the speed the subject had to keep over the first kilometre. Then, the only instruction given was to run as fast as possible until the finish line. They were given distance feedback each kilometre completed. During the three triathlon tests athletes were encouraged to drink $250 \mathrm{~mL}$ after 3, 6 and 9 kilometres.

\section{Measurement of kinematic variables}


1 Running speed was continuously recorded thanks to a s3 accelerometer (Polar RS800sd,

2 Kempele, Finland) (Hausswirth et al. 2009). Three days before the first test, it was calibrated

3 to integrate each runner's stride characteristics, as recommended by the manufacturer.

4 Subjects had to follow a pace close to the speed they would adopt over the control run (i.e.18

$5 \mathrm{~km} \cdot \mathrm{h}^{-1}$ ) for $2 \mathrm{~km}$. They received audio cues via a beeper; the cue rhythm determined the speed

6 needed to cover $20 \mathrm{~m}$.

Measurement of metabolic variables

9 After 35-km of cycling, the subjects were stopped to be equipped with the same portable gas analyser employed during the running pre-test. Thus, the cycle-to-run transition was reduced in time in order to reproduce competition conditions (i.e. 30s) (Millet and Vleck 2000). The physiological data $\left(\dot{\mathrm{V}}_{2}, \dot{\mathrm{V}}_{\mathrm{E}}\right.$ ) were averaged every $5 \mathrm{~s}$ from the breath-by-breath values. They were analysed at the beginning $(0.5-1 \mathrm{~km})$, in the middle $(4.5-5 \mathrm{~km})$ and at the end of each run (9-9.5 km).

\section{Blood sampling}

Blood samples were taken from ear lobes at the end of the cycling phase, after 5-km of running and at the end of the $10-\mathrm{km}$ run for the analysis of blood lactate concentration $\left(\left[\mathrm{La}^{-}\right]_{\mathrm{b}}\right)$ (Lactate Pro, Akray Inc, Kyoto, Japan).

\section{Statistical analyses}


1 All data were expressed as mean \pm standard deviation. A two-way analysis of variance

2 (pacing strategy $\mathrm{x}$ time period) for repeated measures was performed to analyse the effects of

3 the time period and the pace adopted during the first run kilometre using running speed, HR,

$4 \quad \dot{\mathrm{V}}_{2}, \dot{\mathrm{V}}_{\mathrm{E}}$ and $\left[\mathrm{La}^{-}\right]_{\mathrm{b}}$ values as dependent variables. A Newmann-Keuls post hoc test was used

5 to determine differences among all paces and periods during exercise. The level of 6 significance was set at $p<0.05$ for all statistical procedures.

RESULTS

All subjects completed the protocol without problem and remarked that both C-Run and triathlon trials were perceptually similar to competition races.

Training load. No significant difference in training volume and training session distribution in zone1, zone 2 and zone 3 were found between each 10-day period elapsing two tests ( $p=$ 0.97).

Performances. No significant difference was observed between the swimming-cycling phases of the three triathlon sessions ( $p>0.05$, Table 2$)$. There was a systematic significant difference in time required to complete the first $1 \mathrm{~km}$ in relation to the starting strategy (200 \pm 15 s, $190 \pm 14$ s, $210 \pm 17$ s and $220 \pm 18$ s for C-Run, Tri-Run $+5 \%$, Tri-Run-5\% and Tri-Run. $10 \%$, respectively, $p$ ranging from 0.001 to 0.014$)$. Tri-Run $-5 \%$ resulted in a significantly faster overall 10-km run performance than Tri-Run ${ }_{+5 \%}$ and Tri-Run $-10 \%(p=0.005$ and $p=0.02$, 
with Tri-Run $_{+5 \%}$ and Tri-Run $-10 \%$, respectively) but no significant difference with C-Run ( $p=$ 0.58) $\left(2028 \pm 78 \mathrm{~s}\right.$ and $17.8 \pm 0.4 \mathrm{~km} \cdot \mathrm{h}^{-1}$ vs. $2000 \pm 72 \mathrm{~s}$ and $18.0 \pm 0.6 \mathrm{~km} \cdot \mathrm{h}^{-1}, 2178 \pm 121 \mathrm{~s}$ and $16.5 \pm 0.9 \mathrm{~km} \cdot \mathrm{h}^{-1}, 2087 \pm 88 \mathrm{~s}$ and $17.2 \pm 0.6 \mathrm{~km} \cdot \mathrm{h}^{-1}$, for Tri-Run-5\%, C-Run, Tri-Run $+5 \%$ and Tri-Run-10\%, respectively, Table 2).

Physiological parameters. Table 3 indicates mean values for $\mathrm{HR}, \dot{\mathrm{V}} \mathrm{O}_{2}, \dot{\mathrm{V}}_{\mathrm{E}}$ and blood lactate for the running bouts.

Time period effect. $\dot{\mathrm{V}} \mathrm{O}_{2}, \dot{\mathrm{V}}_{\mathrm{E}}$ and $\mathrm{HR}$ at the middle and at the end of the run tended to be lower than their corresponding initial value during $\operatorname{Tri}^{-R{ }+5 \%}(p=0.081$ and $p=0.085$ for $\dot{\mathrm{V}} \mathrm{O}_{2}, p=0.071$ and $p=0.080$ for $\dot{\mathrm{V}}_{\mathrm{E}}, p=0.080$ and $p=0.057$ for HR, when considering km5 and km-10 with km-1, respectively). On the contrary, $\dot{\mathrm{V}}_{\mathrm{E}}$ and HR were significantly higher after 5 and 9.5-km than at the beginning of the run for C-Run, Tri-Run-5\% and Tri-Run-10\% ( $p$ $=0.041$ and $p=0.008$ and $p=0.011$ for $\dot{\mathrm{V}}_{\mathrm{E}}, p=0.035$ and $p=0.003$ and $p=0.006$ for HR, when considering km-5 and km-10 with km-1, for C-Run, Tri-Run-5\% and Tri-Run-10\%, respectively). No significant difference was observed between 5-km [La“ $]_{\mathrm{b}}$ and corresponding initial values excepted for Tri-Run $+5 \%$, whose $\left[\mathrm{La}^{-}\right]_{\mathrm{b}}$ value increased from $2.9 \pm 0.2 \mathrm{mmol}^{-\mathrm{L}^{-1}}$ to $4.9 \pm 0.5 \mathrm{mmol} . \mathrm{L}^{-1}$ during this period $(p=0.035)$. All final $\left[\mathrm{La}^{-}\right]_{\mathrm{b}}$ values for all the runs were significantly higher than their corresponding initial values $(p=0.014, p=0.021$, and $p=$ 0.029, for C-Run, Tri-Run-5\% and Tri-Run-10\%, respectively).

Pacing strategy effect. The statistical analysis indicated a significant effect of pacing strategy on $\dot{\mathrm{V}} \mathrm{O}_{2}, \dot{\mathrm{V}}_{\mathrm{E}}$ and HR during the first kilometre of the running phase $(p<0.05) . \dot{\mathrm{V}} \mathrm{O}_{2}, \dot{\mathrm{V}}_{\mathrm{E}}$ and HR recorded during Tri-Run+5\% after km-1 were indeed significantly higher than C-Run, Tri- 
1 Run $_{-5 \%}$ and Tri-Run $-10 \%\left(p<0.05 \text {, Table 3). On the contrary, } \dot{\mathrm{V}} \mathrm{O}_{2}, \dot{\mathrm{V}}_{\mathrm{E}} \text {, HR and [La }{ }^{-}\right]_{\mathrm{b}}$ during

2 Tri-Run $_{-5 \%}$ were higher than during the three other conditions after 5 and 9.5-km $(p<0.05)$, except $\left[\mathrm{La}^{-}\right]_{\mathrm{b}}$ at km-5 $(p=0.12)$. Tri-Run $+5 \%$ demonstrated greater $\left[\mathrm{La}^{-}\right]_{\mathrm{b}}$ at km-5 than C-Run and the two other triathlon runs $(p=0.031, p=0.033$, and $p=0.038$, when considering C-

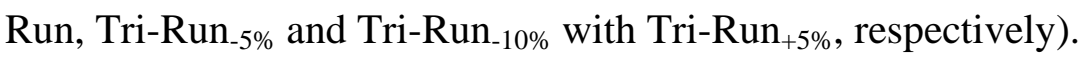

\section{DISCUSSION}

The main finding of this study was that a the best initial pacing strategy during the running leg of a triathlon is to perform the first kilometre $5 \%$ slower than the average pace of a $10-\mathrm{km}$ control run. A 20 sec-variation in running time over the first kilometre led to a significant difference of $150 \pm 21 \mathrm{~s}$ on the 10 -km triathlon run performance. This result is even more relevant considering that the differential time at the finish line between the top 10 triathletes during World Cup triathlons is usually shorter than one minute (Millet and Vleck 2000, Vleck et al. 2006, Vleck et al. 2008, Le Meur et al. 2009). To our knowledge, this study is the first to highlight performance improvements by forcing highly-trained athletes to change their usual pattern of energy expenditure. In previous studies (Hettinga et al 2006, Hettinga et al 2007), such protocol was always associated with performance decrements.

In the present study, triathletes significantly increased their 10-km C-Run time by $1.4 \%, 4.4 \%$ and $8.9 \%$ during Tri-Run $-5 \%$, Tri-Run $-10 \%$ and Tri-Run $+5 \%$, respectively $(p<0.05$, Fig. 2$)$. The 
1 adoption of a fast pace during the first run kilometre induced a significant subsequent

2 slowdown until the $3^{\text {rd }} \mathrm{km}$ (the 9 remaining kilometres were performed $2.7 \pm 0.4 \mathrm{~km} \cdot \mathrm{h}^{-1}$

3 slower than the first one during Tri-Run $+5 \%, p<0.01$; Fig. 2). Conversely, triathletes

4 succeeded in increasing their speed by $1.0 \pm 0.3 \mathrm{~km} \cdot \mathrm{h}^{-1}$ over the same section during Tri-Run. $5 \quad 5 \%$ (Fig. 2).

7 The main explanation to elucidate the weaker performance observed during the Tri-Run $+5 \%$ time-trial is that the pace was centrally down-regulated in a feed-forward manner to avert premature fatigue during exercise, as already proposed by Ulmer (1996). Tucker (2009) has recently proposed that alterations in pacing strategy occur to prevent harmful or catastrophic changes from occurring before the end of exercise, while still optimizing performance. Two major limiting physiological changes may be identified here; of metabolic and ventilatory origins respectively.

The present results revealed a significantly higher metabolic demand during the first kilometre of Tri-Run $_{+5 \%}$ than during Tri-Run-5\% and Tri-Run-10\% (-8.9\% and $-16.7 \%$ concerning $\dot{\mathrm{V}} \mathrm{O}_{2} ;-9.7 \%$ and $-14.5 \%$ concerning $\dot{\mathrm{V}}_{\mathrm{E}}$, for Tri-Run-5\% and Tri-Run-10\%, respectively; $p<0.05$, Table 3). After 5-km, we still observed a greater anaerobic contribution for Tri-Run $+5 \%$ than the 2 other strategies in spite of a significantly lower speed $(4.9 \pm 0.5$ mmol. $\mathrm{L}^{-1}$ and $16.4 \pm 0.4 \mathrm{~km} . \mathrm{h}^{-1}, 3.8 \pm 0.2 \mathrm{mmol} . \mathrm{L}^{-1}$ and $18.0 \pm 0.3 \mathrm{~km} . \mathrm{h}^{-1}, 3.6 \pm 0.4 \mathrm{mmol} . \mathrm{L}^{-}$ ${ }^{1}$ and $16.7 \pm 0.4 \mathrm{~km} \cdot \mathrm{h}^{-1}$ for Tri-Run ${ }_{+5 \%}$, Tri-Run $-5 \%$ and Tri-Run $-10 \%$, respectively; $p<0.05$; Table 3). These results are in accordance with previous studies about pacing strategies in swimming (Thompson et al. 2003), cycling (Foster et al. 1993, Hettinga et al. 2006) and 
running (Billat et al. 2001), which demonstrated that a fast start induces a higher supply of anaerobic pathways to achieve a fixed distance. Moreover, Kreider et al. (1988) explained that the cycle-to-run transition causes a redistribution of blood flow between the different muscular groups involved during running. The delay in the shunting of blood to the upper extremities may then increase the rate of glycolysis in both trunk and arms' muscles. TriRun $_{+5 \%}$ may then have induced higher metabolic disturbances through the overall run than Tri-Run-5\% and Tri-Run-10\%.

Another explanation was that a "negative pacing strategy" (i.e. whereby speed gradually would have increased) would have generated premature respiratory disturbances during the cycle-run transition. At the end of the first kilometre of Tri-Run $+5 \%$, triathletes reached $93.9 \pm$ $6.1 \%$ of $\dot{\mathrm{V}}_{\text {Emax }}$ determined from the laboratory incremental test. These values were significantly higher for Tri-Run $+5 \%$ than Tri-Run $-5 \%$ and Tri-Run-10\% $\left(86.5 \pm 7.8 \%\right.$ of $\dot{\mathrm{V}}_{\text {Emax }}$ and $81.7 \pm 6.9 \%$ of $\dot{\mathrm{V}}_{\text {Emax }}$, for Tri-Run-5\% and Tri-Run-10\%, respectively; $\left.p<0.05\right)$. Hill et al. (1991) demonstrated that the crouched position adopted by triathletes during cycling increases abdominal impedance and diaphragmatic work. Moreover, Boussana et al. (2003) reported that a moderate intensity cycle-to-run combination, not performed to exhaustion induced a decrease in respiratory muscle performance. Another study showed that the respiratory muscle fatigue induced by prior cycling was maintained and not reversed by the subsequent run (Galy et al. 2003). As triathletes here reached higher running intensity than during these studies $\left(87 \% \dot{\mathrm{V}} \mathrm{O}_{2 \max }\right.$ vs. $\left.75 \% \dot{\mathrm{V}} \mathrm{O}_{2 \max }\right)$, Tri-Run $+5 \%$ may have led to greater respiratory disturbances than Tri-Run-5\% and Tri-Run-10\%. 
1 Nevertheless, two major observations led us to hypothesise that the weaker performances observed during Tri-Run $+5 \%$ were mainly due to a reduction of the cognitive drive and not to a peripheral fatigue. Firstly, triathletes succeeded to perform an "end-spurt" in the last kilometre, whereas they showed the typical symptoms of fatigue as indicated by the fall of running speed until the third kilometre (Fig. 2). Tucker (2009) has proposed that the occurrence of an end-spurt indicated that the distribution of pace selected during self-paced exercise is centrally regulated in accordance to an "anticipatory - feedback RPE model”. This final increase in running speed during Tri-Run $+5 \%$ supports the notion that the pacing strategy selected was continuously altered throughout the event, possibly in response to changing afferent signals. It is suggests that exercise demands were somewhat uncertain at commencement of the trial and gradually resolved as the endpoint approached. As a result, running pace was subconsciously attenuated until the last kilometre was reached. As the role of the regulatory processes was to ensure that severe derangements to homeostasis did not occur, this uncertainty may have resulted in the maintenance of a motor unit and metabolic reserve throughout Tri-Run $+5 \%$. From this perspective the weaker performance achieved during Tri-Run $+5 \%$ would have been due primarily to a decrease in motor unit recruitment and not to an effective drastic failure of the ventilatory function or of the homeostasis in the exercising limbs. Moreover, peripheral fatigue results in a progressive decline in force production (Gandevia 2001). In the present experiment, we didn’t observe such a progressive fall in running speed but a sudden slowdown after $\mathrm{km} \mathrm{2.} \mathrm{Speed} \mathrm{decreased} \mathrm{by} 1.5 \mathrm{~km} . \mathrm{h}^{-1}$ per kilometre between kilometre 2 to 4 and only by $0.2 \mathrm{~km} \cdot \mathrm{h}^{-1}$ per kilometre during the 5 subsequent ones (Fig. 2). Thus, we speculated that the adoption of a fast running start may have generated a greater rate of received exertion (RPE) than the one the central controller considered optimal. We hypothesised that triathletes might have been suddenly restrained to slowdown until their RPE returned to a “tolerable” level. 
2 Another interesting finding of our study was that the differential time between Tri-Run $-10 \%$ 3 and Tri-Run $-5 \%$ reached $59 \pm 11$ s at the end of the race, even if the differential time was

4 reached at 10 s at the end of the first kilometre. $\dot{\mathrm{V}} \mathrm{O}_{2}, \dot{\mathrm{V}}_{\mathrm{E}}$ and $\left[\mathrm{La}^{-}\right]_{\mathrm{b}}$ were significantly lower 5 after 5 and 9.5-km for Tri-Run-10\% than Tri-Run-5\%, suggesting that triathletes didn't succeed in reaching the maximal workrate they might have been able to sustain. The reason of this finding remains unclear. Several field-based researches reported that triathletes adopted a high initial pace during the cycle-to-run transition during both competitions (Vleck et al. 2006, Vleck et al. 2008, Le Meur et al. 2009) and multi-transition training sessions (Millet and Vleck 2000). For instance Le Meur et al. (2009) showed that all of the 136 triathletes competing in the 2007 Beijing ITU WC event adopted a "positive pacing strategy" (whereby speed gradually declines, Abbiss and Laursen 2008) through the running phase. During this race, the first of the four laps was run $10.0 \%$ faster than the three remaining laps. Then, we can consider that Tri-Run $+5 \%$ represented the usual strategy experienced by triathletes and that Tri-Run $-10 \%$ was more distant than Tri-Run-5\% from triathletes' usual starting strategy. Over the first kilometre of Tri-Run-5\% triathletes were forced to start 20 seconds slower than they used to (i.e. Tri-Run $+5 \%$ ), whereas this differential starting time reached 35 seconds during

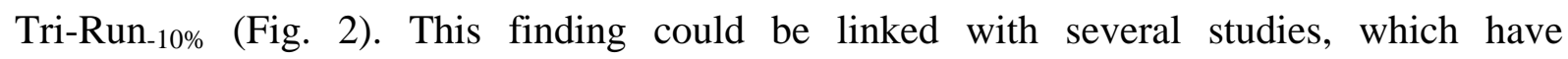
demonstrated that the pacing strategy is influenced by prior experience (Ansley et al 2004, Mauger et al., 2009, Micklewright et al. 2009, Foster et al. 2009). A recent research conducted by Foster et al. (2009) demonstrated that the pattern of energy expenditure during time trial exercise appears to follow a predetermined template associated with prior experience, which is modified by a variety of sensory feedbacks mechanisms. From this perspective, Tri-Run-10\% may have been more disturbing for triathletes than Tri-Run-5\% by 
1 providing more atypical internal feedbacks than those they usually perceived during the cycle-

2 to-run transition (see physiological responses in Table 3). The present results suggested that

3 the higher the sensory feedbacks are modified comparing to prior experience, the more triathletes had difficulties to adjust their pace. We speculated that triathletes would have taken benefits particularly from further experimentations of Tri-Run-10\% strategy to improve their ability to adjust quickly and to maintain an optimal pace after a slow first kilometre. Indeed Mauger et al. (2009) have demonstrated recently that cyclists completed a time-trial closed to their personal best -without any external feedbacks - only if previous experience (i.e. at least 4 time trials) has been gained to develop the appropriate optimal strategy. Similarly, Foster et al. (2009) showed that the "anticipatory-feedback RPE model” is not a non-constant feature and may require some time to fully develop. Further studies are required to confirm this hypothesis.

In conclusion, this study demonstrated that elite triathletes should slightly reduce their freelychosen pace over the first run kilometre of short distance triathlons. The present results showed that pacing during the cycle-to-run transition is crucial for the development of the running phase as a whole. In this context, the best running strategy following cycling is to perform the first kilometre 5\% slower than the average speed of a 10-km control run. Highlytrained triathletes would benefit to automate this particular pace during back to back cycle-run training as both slower and higher initial running speed led to weaker performance. Considering the high correlation systematically reported between finish position and running performance during ITU World Cup races for both sexes (Vleck et al. 2006, Vleck et al. 2008, Le Meur et al. 2009), pacing might be the main factor in improving the running performance achieved in competition by world-class triathletes. 


\section{Acknowledgments}

2 This study was made possible by technical support from the French National Institute of Sport

3 and Physical Education, the French Federation of Triathlon and the University of South

4 Toulon-Var. The authors are especially grateful to the athletes and coaches for their help and

5 cooperation. They also gratefully acknowledge Jessica Harrison for her help in the correction

6 of this manuscript.

7

8 


\section{Conflict of interest}

2 The authors declare that they have no conflict of interest.

3

\section{Ethical standards}

5 The subjects were fully informed of the content of the experiment, and written consent was

6 obtained before any testing, according to local ethical committee guidelines (Saint Germain

7 en Laye, France).

8 


\section{REFERENCES}

Abbiss CR, Laursen PB (2008) Describing and understanding pacing strategies during athletic competition. Sports Med 38:239-52

Ansley L, Robson PJ, St Clair Gibson A, Noakes TD (2004) Anticipatory pacing strategies during supramaximal exercise longer than 30 s. Med Sci Sports Exerc 36:309-14

Beaver WL, Wasserman K, Whipp BJ (1986) A new method for detecting anaerobic threshold by gas exchange. J Appl Physiol 60:2020-7

Bentley DJ, Millet GP, Vleck VE, McNaughton LR (2002) Specific aspects of contemporary triathlon. Sports Med 32:345-359

Bernard T, Vercruyssen F, Mazure C, Gorce P, Hausswirth C, Brisswalter J (2007) Constant versus variable-intensity during cycling: effects on subsequent running performance. Eur J Appl Physiol 99:103-11

Bernard T, Hausswirth C, Le Meur Y, Bignet F, Dorel S, Brisswalter J (2009) Distribution of power output during the cycling stage of a triathlon World Cup. Med Sci Sports Exerc 41:1296-302

Billat VL, Slawinski J, Danel M, Koralsztein JP (2001) Effect of free versus constant pace on performance and oxygen kinetics in running. Med Sci Sports Exerc 33:2082-8

Boussana A, Matecki S, Galy O, Hue O, Ramonatxo M, Le Gallais D (2001) The effect of exercise modality on respiratory muscle performance in triathletes. Med Sci Sports Exerc 33:2036-43

Esteve-Lanao J, Foster C, Seiler S, Lucia A (2007) Impact of training intensity distribution on performance in endurance athletes. J Strength and Cond Res 21:943-9 
Fiskestrand A, Seiler KS (2004) Training and performance characteristics among

2 Norwegian international elite rowers 1970-2001. Scand J Med Sci Sports 14:303-10

Foster C, Snyder AC, Thompson NN, Green MA, Foley M, Schrager M (1993) Effect of pacing strategy on cycle time trial performance. Med Sci Sports Exerc 25:383-8

Foster C, Hendrickson K J, Peyer K, Reiner B, de Koning JJ, Lucia A, Battista RA, Hettinga FJ, Porcari JP, Wright G (in press) Pattern of developing the performance template. Br J Sports Med

Galy O, Hue O, Boussana A, Peyreigne C, Couret I, Le Gallais D, Mercier J, Préfaut C (2003) Effects of the order of running and cycling of similar intensity and duration on pulmonary diffusing capacity in triathletes. Eur J Appl Physiol 90:489-85

Gandevia SC (2001) Spinal and supraspinal factors in human muscle fatigue. Physiol Rev 81:1725-89

Gottshall JS, Palmer BM (2002) The acute effects of prior cycling cadence on running performance and kinematics. Med Sci Sports Exerc 34:1518-22

Hausswirth C, Bigard AX, Berthelot M, Thomaïdis M, Guezennec CY (1996) Variability in energy cost of running at the end of a triathlon and a marathon. Int J Sports Med $17: 572-9$

Hausswirth C, Bigard AX, Guezennec CY (1997) Relationships between running mechanics and energy cost of running at the end of a triathlon and a marathon. Int J Sports Med 18:330-9

Hausswirth C, Lehénaff D, Dréano P, Savonen K (1999) Effects of cycling alone or in a sheltered position on subsequent running performance during a triathlon. Med Sci Sports Exerc 31:599-604 
2 (2001) Effect of two drafting modalities in cycling on running performance. Med Sci Sports Exerc 33:485-92

Hausswirth C, Brisswalter J (2008) Strategies for improving performance in long duration events: Olympic distance triathlon. Sports Med 38:881-91

Hausswirth C, Le Meur Y, Couturier A, Bernard T, Brisswalter J (2009) Accuracy and repeatability of the Polar ${ }^{\circledR}$ RS800sd to evaluate stride rate and running speed. Int J Sports Med 30:354-9

Hettinga FJ, deKoning JJ, Broersen FT, Van Geffen P, Foster C (2006) Pacing strategy and the occurrence of fatigue in 4000-m cycling time trials. Med Sci Sports Exerc 38:1484-91

Hettinga FJ, deKoning JJ, Meijer E, Teunissen L, Foster C (2007) The effect of pacing strategy on energy expenditure during a 1500-m cycling time trial. Med Sci Sports Exerc 39:2212-18

Hill NS, Jacoby C, Farber W (1991) Effect of an endurance triathlon on pulmonary function. Med Sci Sports Exerc 23:1260-1264

Howley ET, Bassett DR Jr, Welch HG (1995) Criteria for maximal oxygen uptake: review and commentary. Med Sci Sports Exerc 27:1292-301

Kreider RB, Cundiff DE, Hammett JB, Cortes CW, William KW (1988) Effect of cycling on running performance in triathletes. Annals of Sports Med 3:220-225

Le Meur Y, Hausswirth C, Dorel S, Bignet F, Brisswalter J, Bernard T (2009) Influence of gender on pacing adopted by elite triathletes during a competition. Eur J Appl Physiol $106: 535-45$ 
Mauger AR, Jones AM, Williams CA (2009) Influence of feedback and prior experience on pacing during a 4-km cycle time trial. Med Sci Sports Exerc 41:451-58

Micklewright D, Papadopoulou E, Swart J Dr, Noakes TD (in press) Previous experience influences pacing during 20-km time trial cycling. Br J Sports Med

Millet GP, Vleck VE (2000) Physiological and biomechanical adaptations to the cycle to run transition in Olympic triathlon: review and practical recommendations for training. $\mathrm{Br} \mathrm{J}$ Sports Med 34:384-90

Seiler KS, Kjerland GO (2006) Quantifying training intensity distribution in elite endurance athletes: Is the evidence for an 'optimal' distribution ? Scand J Med Sci Sports $16: 49-56$

Thompson KG, MacLaren DP, Lees A, Atkinson G (2003) The effect of even, positive and negative pacing on metabolic, kinematic and temporal variables during breaststroke swimming. Eur J Appl Physiol 88:438-43

Tucker R (2009) The anticipatory regulation of performance: The physiological basis for pacing strategies and the development of a perception-based model for exercise performance. Br J Sports Med 43:392-400

Tucker R, Noakes TD (2009b) The physiological regulation of pacing strategy during exercise: a critical review. Br J Sports Med 43:e1

Ulmer HV (1996) Concept of an extracellular regulation of muscular metabolic rate during heavy exercise in humans by psychophysiological feedback. Experientia 52:416-20 
2 selection affects metabolic responses during cycling and subsequent running time to fatigue.

$3 \quad$ Br J Sports Med 2002;39:267-72

$4 \quad$ Vleck VE, Bürgi A, Bentley DJ (2006) The consequences of swim, cycle, and run 5 performance on overall result in elite Olympic distance triathlon. Int J Sports Med 27:43-8

6 Vleck VE, Bentley DJ, Millet GP, Bürgi A (2008) Pacing during an elite Olympic 7 distance triathlon: comparison between male and female competitors. J Sci Med Sport 11:424$8 \quad 432$

9 
1 FIGURE 1. Graphic representation of the three triathlon conditions and the control run. BS:

2 blood samples. Dark portions represent $\dot{\mathrm{V}}_{2}$ and $\dot{\mathrm{V}}_{\mathrm{E}}$ interval measurements. Tri-Run $+5 \%$, Tri-

3 Run $_{-5 \%}$, Tri-Run-10\% represent triathlon, whose first run kilometre was done 5\% faster, 5\%

4 slower and 10\% slower than the control run (C-Run). The 9 remaining kilometres were left 5 free.

7 FIGURE 2 . Group mean $( \pm \mathrm{SD})$ values for average running speeds $\left(\mathrm{km} . \mathrm{h}^{-1}\right)$ recorded every 8 kilometer during the $10-\mathrm{km}$ run for the Triathlon Runs where the $1^{\text {st }} \mathrm{km}$ was done 9 alternatively 5\% faster (Tri-Run $+5 \%$ ), 5\% slower (Tri-Run $-5 \%$ ) and $10 \%$ slower (Tri-Run $-10 \%$ ) 10 than the Control Run (C-Run): the 9 remaining kilometers were then free in all situations.

11 All values for the Tri-Run $+5 \%$ were significantly different from the corresponding imposed 12 initial pace (excepted for km-2 value), $p<0.01$.

13 All values for the Tri-Run-10\% were significantly different from the corresponding imposed 14 initial pace (excepted for $\mathrm{km}-5$ value), $p<0.01$

15 \$ Significantly different within both Tri-Run $+5 \%$ and Tri-Run $-10 \%$, and within Tri-Run $+5 \%$ and Tri-Run-5\%, $p<0.05$

* Significantly different within both Tri-Run $-5 \%$ and Tri-Run-10\%, and within Tri-Run $-5 \%$ and Tri-Run $+5 \%, p<0.05$ 


\begin{tabular}{|c|c|}
\hline Age (years) & $24 \pm 3$ \\
\hline Height (cm) & $178 \pm 5$ \\
\hline Weight (kg) & $68.2 \pm 6.7$ \\
\hline Swimming training $\left(\mathrm{km}\right.$ week $\left.^{-1}\right)$ & $12.5 \pm 1.9$ \\
\hline Cycling training $\left(\mathrm{km}^{2} \mathrm{week}^{-1}\right)$ & $220 \pm 42$ \\
\hline Running training $\left(\mathrm{km} \mathrm{week}^{-1}\right)$ & $65 \pm 12$ \\
\hline Running $\dot{\mathrm{V}} \mathrm{O}_{2 \max }\left(\mathrm{mL} \cdot \mathrm{min}^{-1} \cdot \mathrm{kg}^{-1}\right)$ & $69.1 \pm 7.1$ \\
\hline Running $\dot{\mathrm{V}}_{\text {Emax }}\left(\mathrm{L} \cdot \mathrm{min}^{-1}\right)$ & $184 \pm 21$ \\
\hline Running $\mathrm{HR}_{\max }\left(\right.$ beats.min ${ }^{-1}$ ) & $194 \pm 7$ \\
\hline
\end{tabular}

Table 1. Characteristics of the subjects participating in the present study $(n=10)$.

Values are expressed as mean $\pm \mathrm{SD}$.

$\dot{\mathrm{V}} \mathrm{O}_{2 \max }$, maximal oxygen uptake; $\dot{\mathrm{V}}_{\text {Emax }}$, maximal minute ventilation; $\mathrm{HR}_{\max }$ : maximal heart rate. 


\begin{tabular}{|c|c|c|c|c|}
\hline$\underbrace{\text { Perf. }}_{\text {Conditions }}$ & $\begin{array}{c}\text { Swimming time } \\
\text { (sec) }\end{array}$ & $\begin{array}{c}\text { Cycling time } \\
\text { (sec) }\end{array}$ & $\begin{array}{l}\text { Running time } \\
\text { (sec) }\end{array}$ & $\begin{array}{c}\text { Overall time } \\
\text { (sec) }\end{array}$ \\
\hline C-Run & & & $2000 \pm 72^{\mu \mu £ £}$ & \\
\hline Tri-Run $+5 \%$ & $1278 \pm 54$ & $4260 \pm 52$ & $2178 \pm 121^{* * \$ \$ \mu \mu}$ & $7716 \pm 196^{\$ \$ \mu \mu}$ \\
\hline Tri-Run-5\% & $1275 \pm 51$ & $4255 \pm 50$ & $2028 \pm 78^{£ £ \mu}$ & $7558 \pm 188^{£ £ \mu}$ \\
\hline Tri-Run-10\% & $1281 \pm 52$ & $4263 \pm 57$ & $2087 \pm 88^{\$ * £}$ & $7631 \pm 191^{\$ £}$ \\
\hline
\end{tabular}

Table 2. Overall and isolated performances achieved during the three triathlons. Values are expressed as mean $\pm \mathrm{SD}$.

Significantly different from C-Run group, $* p<0.05, * * p<0.01$

Significantly different from Tri-Run ${ }_{+5 \%}, £ p<0.05$, $£ £ p<0.01$

Significantly different from Tri-Run-5\%, $\$ p<0.05, \$ \$ p<0.01$

Significantly different from Tri-Run ${ }_{-10 \%}, \mu p<0.05, \mu \mu p<0.01$ 


\begin{tabular}{|c|c|c|c|c|c|c|c|c|c|c|c|c|}
\hline Param. & \multicolumn{3}{|c|}{$\begin{array}{l}\text { Oxygen uptake } \\
\left(\begin{array}{c}\left(\mathrm{V}_{2}\right) \\
\left.{ }^{1} \cdot \mathrm{kg}^{-1}\right)\end{array}\right.\end{array}$} & \multicolumn{3}{|c|}{$\begin{array}{l}\text { Expiratory flow } \\
\left(\dot{\mathrm{V}}_{\mathrm{E}}\right)\left(\mathrm{L}_{\mathrm{min}}{ }^{-1}\right)\end{array}$} & \multicolumn{3}{|c|}{$\begin{array}{c}\text { Heart rate (HR) } \\
\text { (beats.min }{ }^{-1} \text { ) }\end{array}$} & \multicolumn{3}{|c|}{$\begin{array}{l}\text { Blood lactate } \\
\quad\left(\left[\mathrm{La}^{-}\right]_{\mathrm{b}}\right) \\
\left(\mathrm{mmol} \cdot \mathrm{L}^{-1}\right)\end{array}$} \\
\hline $\begin{array}{c}\text { Distance } \\
(\mathbf{k m})\end{array}$ & $\begin{array}{l}0.5 \\
-1\end{array}$ & $\begin{array}{c}4.5- \\
5\end{array}$ & $\begin{array}{c}9- \\
9.5\end{array}$ & $\begin{array}{c}0.5- \\
1\end{array}$ & $\begin{array}{c}4.5- \\
5\end{array}$ & $\begin{array}{c}9- \\
9.5\end{array}$ & $0.5-1$ & $4.5-5$ & $9-9.5$ & $\mathbf{0}$ & 5 & 10 \\
\hline C-Run & $\begin{array}{c}61.5 \\
\pm 5.1 \\
\$\end{array}$ & $\begin{array}{l}60.7 \\
\pm 5.8 \\
\$ £\end{array}$ & $\begin{array}{l}64.4 \\
\pm 5.7 \\
£ \$ \$\end{array}$ & $\begin{array}{c}173 . \\
2 \pm \\
12.3 \\
\$\end{array}$ & $\begin{array}{c}179 . \\
8 \pm \\
14.3 \\
\$ \$ £\end{array}$ & $\begin{array}{c}182 . \\
1 \pm \\
9.5 \\
\\
\$ \$ £\end{array}$ & $\begin{array}{c}176.2 \\
\pm \\
17.3 \\
E\end{array}$ & $\begin{array}{c}186.2 \\
\pm \\
17.9 \\
\\
\$\end{array}$ & $\begin{array}{c}189.3 \\
\pm \\
18.2 \\
\$ \$\end{array}$ & $\begin{array}{c}0.8 \\
\pm \\
0.3 \\
\$ \$ \\
£\end{array}$ & $\begin{array}{c}3.2 \\
\pm \\
0.4 \\
\$\end{array}$ & $\begin{array}{c}3.4 \\
\pm \\
0.3 \\
£\end{array}$ \\
\hline Tri-Run $_{+5 \%} \%$ & $\begin{array}{l}66.1 \\
\pm 7.0 \\
* £\end{array}$ & $\begin{array}{l}54.9 \\
\pm 6.8 \\
* £ £\end{array}$ & $\begin{array}{l}55.1 \\
\pm 6.7 \\
* * \\
\\
\text { } £\end{array}$ & $\begin{array}{c}182 . \\
1 \pm \\
9.2 \\
*\end{array}$ & $\begin{array}{c}160 . \\
5 \pm \\
12.3 \\
* * £ £\end{array}$ & $\begin{array}{c}162 . \\
1 \pm \\
13.4 \\
* * £\end{array}$ & $\begin{array}{c}186.0 \\
\pm \\
16.5 \\
*_{£ £}\end{array}$ & $\begin{array}{c}176.1 \\
\pm \\
14.8 \\
£^{*}\end{array}$ & $\begin{array}{c}171.6 \\
\pm \\
12.8 \\
* * £ £\end{array}$ & $\begin{array}{c}2.9 \\
\pm \\
0.2 \\
* \\
*\end{array}$ & $\begin{array}{c}.9 \\
\pm \\
0.5 \\
*_{£}\end{array}$ & $\begin{array}{c}4.1 \\
\pm \\
0.3 \\
£\end{array}$ \\
\hline Tri-Run-5\% & $\begin{array}{c}60.9 \\
\pm 5.9 \\
\$\end{array}$ & $\begin{array}{l}65.3 \\
\pm 5.8 \\
* \$ \$\end{array}$ & $\begin{array}{l}68.1 \\
\pm 6.8 \\
* \$ \$\end{array}$ & $\begin{array}{r}167 \\
8 \pm \\
8.2\end{array}$ & $\begin{array}{c}187 . \\
9 \pm \\
11.2 \\
* \$\end{array}$ & $\begin{array}{c}191 . \\
5 \pm \\
10.8 \\
* \$\end{array}$ & $\begin{array}{c}168.0 \\
\pm \\
11.2 \\
* \$\end{array}$ & $\begin{array}{c}190.1 \\
\pm \\
14.2 \\
\\
\$\end{array}$ & $\begin{array}{c}192.8 \\
\pm \\
12.8 \\
\$ \$\end{array}$ & $\begin{array}{c}3.2 \\
\pm \\
0.3 \\
*\end{array}$ & $\begin{array}{c}3.8 \\
\pm \\
0.2 \\
\$\end{array}$ & $\begin{array}{c}5.4 \\
\pm \\
0.4 \\
* \$\end{array}$ \\
\hline Tri-Run $_{-10 \%}$ & $\begin{array}{c}55.1 \\
\pm 4.9 \\
* \$ \$ \\
£\end{array}$ & $\begin{array}{l}57.9 \\
\pm 4.4 \\
\$ £ £\end{array}$ & $\begin{array}{l}60.6 \\
\pm 5.7 \\
* £ \$\end{array}$ & $\begin{array}{c}158 . \\
5 \pm \\
10.8 \\
* \$ £\end{array}$ & $\begin{array}{c}170 . \\
4 \pm \\
11.2 \\
*_{£ £ \$}\end{array}$ & $\begin{array}{c}164 . \\
2 \pm \\
12.1 \\
*_{£ £}\end{array}$ & $\begin{array}{c}159.0 \\
\pm \\
11.3 \\
* * £ \$ \$\end{array}$ & $\begin{array}{c}181.1 \\
\pm \\
17.8 \\
£\end{array}$ & $\begin{array}{c}180.1 \\
\pm \\
17.2 \\
* £ \$\end{array}$ & $\begin{array}{c}3.1 \\
\pm \\
0.2 \\
* \\
*\end{array}$ & $\begin{array}{c}3.6 \\
\pm \\
0.4 \\
\$\end{array}$ & $\begin{array}{c}4.0 \\
\pm \\
0.4 \\
£\end{array}$ \\
\hline
\end{tabular}

Table 3. Group mean ( \pm SD) values for oxygen uptake, expiratory flow, heart rate and blood lactate obtained during the run sessions.

All $\dot{\mathrm{V}} \mathrm{O}_{2}, \dot{\mathrm{V}}_{\mathrm{E}}$ and HR values for both Tri-Run $-5 \%$ and Tri-Run $+5 \%$ were significantly different from the corresponding initial value, $p<0.05$. 
All $\dot{\mathrm{V}}_{\mathrm{E}}$ and HR values for both C-Run and Tri-Run-10\% were significantly different from the corresponding initial value, $p<0.05$

All final $\left[\mathrm{La}^{-}\right]_{\mathrm{b}}$ values for all Runs were significantly different from the corresponding initial value, $p<0.05$

The $5-\mathrm{km}\left[\mathrm{La}^{-}\right]_{\mathrm{b}}$ values for Tri-Run $+5 \%$ were significantly different from the corresponding initial value, $p<0.05$

Significantly different from C-Run group, ${ }^{*} p<0.05$, ${ }^{* *} p<0.01$

Significantly different from Tri-Run $+5 \%$ group, $\$ p<0.05$, $\$ \$ p<0.01$

Significantly different from Tri-Run-5\% group, $£ p<0.05$, $£ £ p<0.01$ 
Triathlons: 3 modalities of running (T-Run ${ }_{+5 \%}$, T-Run. ${ }_{-5 \%}$ and T-Run ${ }_{-10 \%}$ )

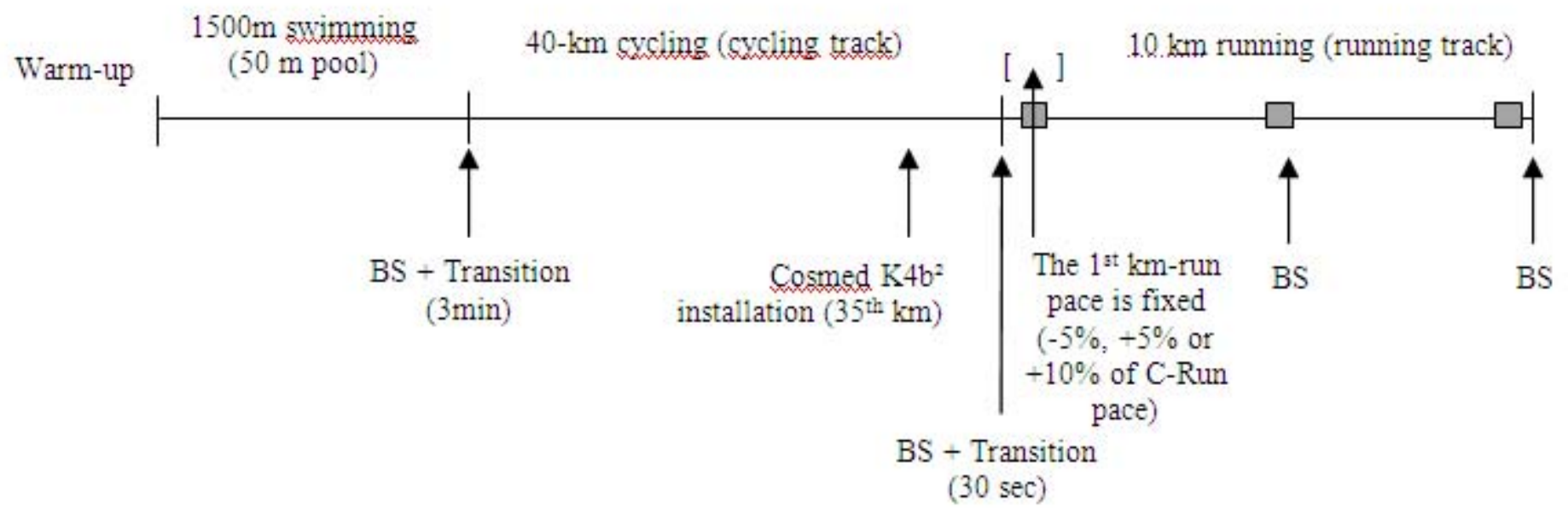

10-km Control Run (C-Run)

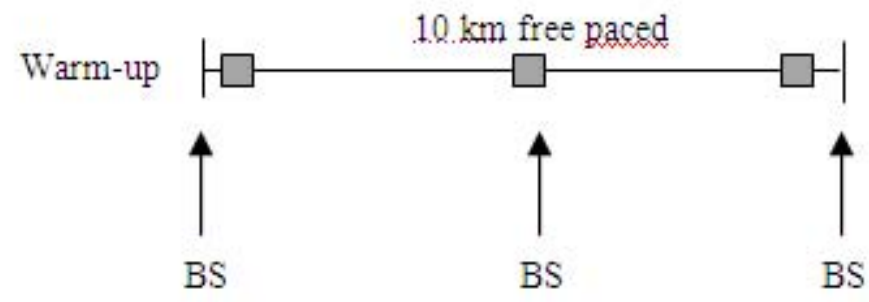




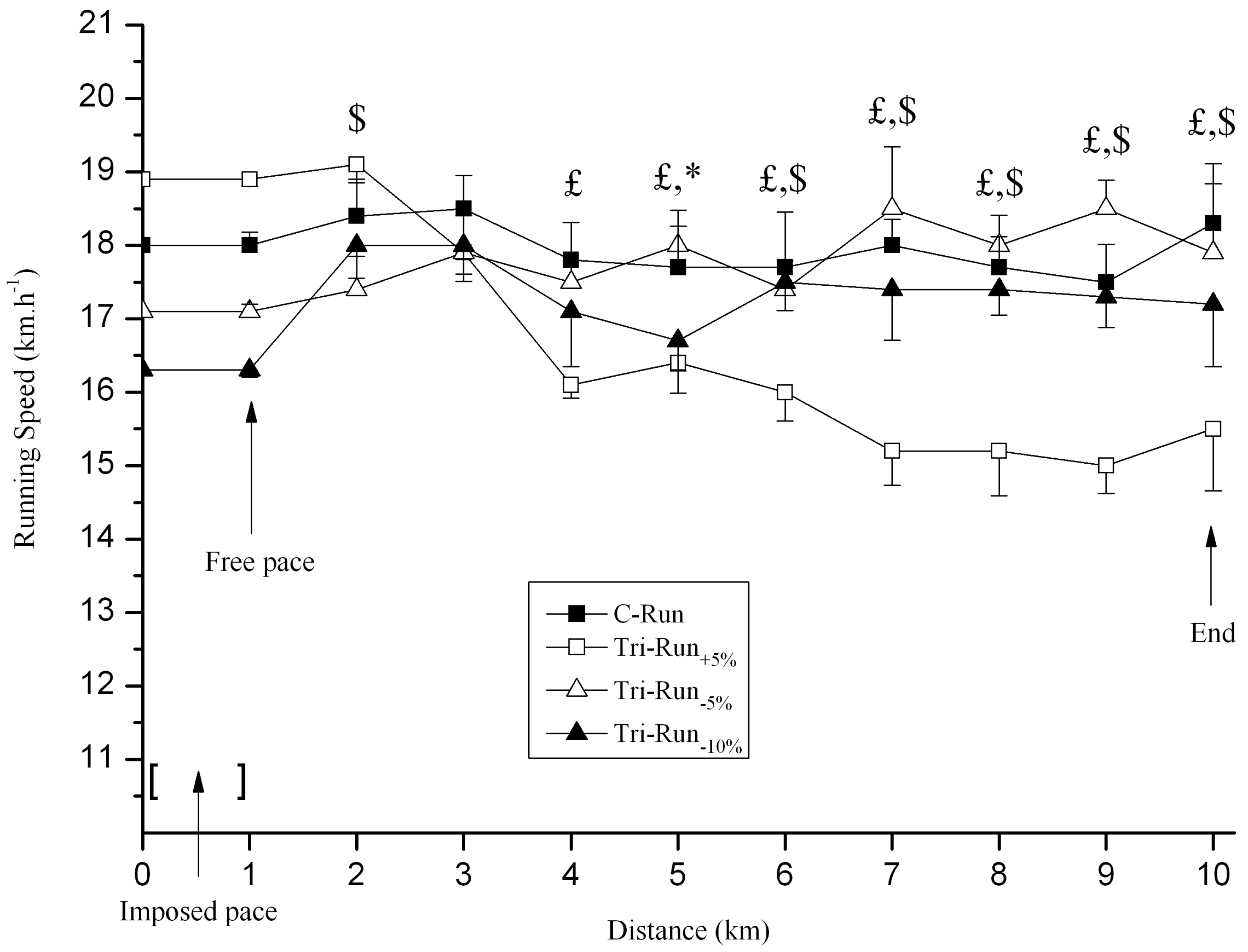

